\title{
Nonlinear Modal Analysis of Structural Systems Using Multi-Mode Invariant Manifolds
}

\author{
ERIC PESHECK, NICOLAS BOIVIN, and CHRISTOPHE PIERRE* \\ Department of Mechanical Engineering and Applied Mechanics, University of Michigan, \\ Ann Arbor, MI 48109-2125, U.S.A. \\ STEVEN W. SHAW \\ Department of Mechanical Engineering, Michigan State University, East Lansing, MI 48824-1226, U.S.A.
}

(Received: 8 February 1999; accepted: 10 August 1999)

\begin{abstract}
In this paper, an invariant manifold approach is introduced for the generation of reduced-order models for nonlinear vibrations of multi-degrees-of-freedom systems. In particular, the invariant manifold approach for defining and constructing nonlinear normal modes of vibration is extended to the case of multi-mode manifolds. The dynamic models obtained from this technique capture the essential coupling between modes of interest, while avoiding coupling from other modes. Such an approach is useful for modeling complex system responses, and is essential when internal resonances exist between modes. The basic theory and a general, constructive methodology for the method are presented. It is then applied to two example problems, one analytical and the other finiteelement based. Numerical simulation results are obtained for the full model and various types of reduced-order models, including the usual projection onto a set of linear modes, and the invariant manifold approach developed herein. The results show that the method is capable of accurately representing the nonlinear system dynamics with relatively few degrees of freedom over a range of vibration amplitudes.
\end{abstract}

Keywords: Normal modes, modal analysis, nonlinear vibration, invariant manifolds.

\section{Introduction}

The concept of normal modes of motion is well developed for linear oscillatory systems, due to the special features of the linear differential equations governing their dynamics. These features allow for the definition of normal modes in terms of eigenvectors (or eigenfunctions) and the expression of an arbitrary system response as a superposition of modal responses [1]. In particular, given the invariance of the normal modes, truncation procedures have been developed to allow for the reduction of the number of modeled (i.e., simulated) modes, and yet for the elimination, in many cases, of contamination from the non-modeled modes.

Many themes present in linear systems may be extended to nonlinear systems. For example, much work has been done on the existence and stability of normal modes of motion for two-degrees-of-freedom, conservative systems $[2,3]$, as well as continuous systems $[4,5]$. However, new methodologies have been developed [6-8] that generalize these definitions to a very wide class of systems which include non-conservative, gyroscopic, and infinitedimensional systems. Essentially, these works define normal modes in terms of motions which occur on low - typically two - dimensional invariant manifolds in the system's phase space. Such motion must be inherently like that of a lower dimensional system, as is desired for a

^ Author for correspondence. Phone: (734) 936-0401, fax: (734) 647-3170, E-mail: pierre@engin.umich.edu. 
normal mode. A constructive technique for generating such manifolds in terms of asymptotic series, without having to solve the equations of motion, is provided by a simple generalization of the method used in constructing approximate center manifolds in bifurcation theory [9]. Using this approach, it is possible to determine the manifolds which represent the normal modes for weakly nonlinear systems. The equations of motion restricted to these manifolds then provide the dynamics of the associated normal modes. The planes tangent to the manifolds at the equilibrium point are the planes on which the usual modal dynamics of the linearized system take place, i.e., they are the familiar eigenspaces. By definition, these nonlinear normal mode manifolds are invariant, such that any motion starting exactly in one nonlinear normal mode will be comprised only of that one for all time and will not generate any motion in the other nonlinear normal modes. On the contrary, a standard linear modal analysis of the system's dynamics - obtained by projection of the equations of motion onto the linear modes - necessarily results in the exchange of energy, or contamination, between linear modes, due to the nonlinear coupling terms between the obtained equations. As was demonstrated previously [10], this may yield inaccurate results if one includes only a few linear modes, or expensive solutions if one includes many of them. Many of the principles inherent to this approach have been modified and/or extended to achieve various ends [11-16], but the basic ideas are outlined in Section 2.

Just as the primary use for normal modes of motion of linear systems is the modal analysis associated with them, the concept of nonlinear normal modes of vibration suggests the definition of a proper 'nonlinear modal analysis' in order to obtain the response of a system in terms of nonlinear modal coordinates. Moreover, one ought to be able to perform model reductions using the nonlinear modal coordinates - as is done for linear systems - which requires the development of efficient truncation procedures, the ultimate goal being to use fewer nonlinear modes than linear ones to perform equally accurate modal analyses of nonlinear systems.

Other works have been done which investigate coupled normal mode motions. Many of these have concentrated on the phenomenon of internal resonance, and have hence made certain restrictions on the candidate systems, and the dynamics therein [2,17]. Another approach [18], similar in spirit to that which follows, uses an action-angle transformation to create 'multi-modes', whose manifolds are then determined numerically. The present paper seeks to extend the invariant manifold-based nonlinear normal mode method, such that practical analysis of larger systems, including those with internal resonances, may be conducted.

Given the definition of the nonlinear normal modes in terms of two-dimensional invariant manifolds, it is clear that (1) they will not interact during a pure modal motion, and (2) they are bound to interact during more general motions. As ignoring these interactions has been found to yield reduced-order models of insufficient accuracy [19], it is essential for any nonlinear extension of modal analysis to account for them in a rigorous manner.

Consequently, a new formulation has been developed to ensure the invariance of the set of modeled nonlinear modes with respect to the non-modeled ones. This formulation, described in Section 3, builds on ideas introduced in [20-22] and generalizes the individually invariant nonlinear normal mode manifolds to multi-mode invariant manifolds. A multi-mode manifold is of dimension $2 M$ when $M$ nonlinear modes are modeled, and includes the influence of all of the $M$ individual nonlinear manifolds defined previously. The interactions between the various modeled nonlinear modes are accounted for at the first stage of the definition process, thus eliminating the need for later work to recover them. The generation of a multi-mode invariant manifold follows closely that of an individually invariant manifold, and approximations for weakly nonlinear systems can be constructed easily using this method. As with the individual 
nonlinear modes, the modes constituting a multi-mode manifold do not interact with the nonmodeled ones for motions occurring on that manifold, thus eliminating contamination from the non-modeled modes.

Closed form solutions have been generated for a subset of nonlinear structural systems with arbitrary quadratic and cubic coupling between their linear modes. This allows for the automated generation of third-order accurate multi-mode manifolds which govern the dynamics of a chosen modal subset. These high-dimensional manifolds may then be used to produce reduced equations of motion for a wide variety of structural systems.

Reduced-order models have been obtained for two continuous systems with discrete nonlinearities, one analytic, and the other finite-element based. These results illustrate the benefits of the formulation compared to classical linear modal analyses of nonlinear systems (i.e., projections of equations of motion onto the linear modes). The dynamics recovered by the multi-mode manifold methodology are generally more accurate than those obtained by a linear modal analysis using the same number of linear modes. It should be noted that strong nonlinearities may require higher-order expansions than those employed herein in order to depict the associated manifold accurately. However, for nonlinearities of sufficiently modest degree, this model reduction method may yield significant computational savings.

\section{Individual Nonlinear Normal Modes}

The equations of motion for the structural systems considered are assumed to be of the form

$$
\left\{\begin{array}{l}
\dot{x}=y_{i}, \\
\dot{y}_{i}=f_{i}\left(x_{1}, \ldots, x_{N}, y_{1}, \ldots, y_{N}\right),
\end{array} \quad i=1, \ldots, N\right.
$$

or

$$
\mathbf{z}=\mathbf{A}(\mathbf{z})
$$

with $\mathbf{z}^{T}=\left[x_{1}, y_{1}, \ldots, x_{N}, y_{N}\right]^{T}$ and $\mathbf{A}(\mathbf{z})^{T}=\left[y_{1}, f_{1}, \ldots, y_{N}, f_{N}\right]^{T}$ (where ${ }^{T}$ denotes a transpose), where any necessary discretization has been achieved previously, for instance using the modes of the linearized system. In the case of a discretized continuous system, $\mathbf{x}^{T}=\left[x_{1}, \ldots, x_{N}\right]^{T}$ and $\mathbf{y}^{T}=\left[y_{1}, \ldots, y_{N}\right]^{T}$ would represent some assumed modal coordinates and velocities, while for discrete systems they would represent generalized coordinates (displacements or rotations) and the corresponding generalized velocities. Furthermore, $\mathbf{f}^{T}=$ $\left[f_{1}, \ldots, f_{N}\right]^{T}$ represents some nonlinear, autonomous forcing on the system.

For a nonlinear, autonomous, oscillatory system such as that defined above, a normal mode of motion is a motion which takes place on a two-dimensional invariant manifold in the system's phase-space. This manifold passes through a stable equilibrium point of the system $(\mathbf{x}, \mathbf{y})=(0,0)$ and it is tangent to an eigenspace of the system linearized about that equilibrium [6]. This invariant manifold and the dynamics on it can be described by a pair of independent coordinates, which can be chosen to be a single displacement-velocity pair. However, this procedure must be modified for certain degenerate cases [6,7], and internal resonances $[20,2]$. For the $k$ th nonlinear normal mode, it is a natural choice to define $u_{k}=x_{k}$ 
and $v_{k}=y_{k}$, so that all displacements and velocities can be related to $\left(u_{k}, v_{k}\right)$ only - thus enforcing the two-dimensionality and the invariance of the motion - as

$$
\left\{\begin{array}{l}
x_{i}=X_{i}\left(u_{k}, v_{k}\right), \\
y_{i}=Y_{i}\left(u_{k}, v_{k}\right) .
\end{array} \quad i=1, \ldots, N, \quad i \neq k,\right.
$$

Substitution into the equations of motion yields a set of constraint equations which describe the geometry of the nonlinear invariant manifold, as

$$
\left\{\begin{array}{l}
\frac{\partial X_{i}}{\partial u_{k}} v_{k}+\frac{\partial X_{i}}{\partial v_{k}} f_{k}=Y_{i}, \\
\frac{\partial Y_{i}}{\partial u_{k}} v_{k}+\frac{\partial Y_{i}}{\partial v_{k}} f_{k}=f_{i},
\end{array} \quad i=1, \ldots, N, \quad i \neq k,\right.
$$

where use has been made of the $k$ th pair of equations of motion, i.e., $\dot{u}_{k}=v_{k}$ and $\dot{v}_{k}=f_{k}$. Notice no assumption has yet been made on $u_{k}$ and $v_{k}$, and therefore Equation (4) describes the $k$ th nonlinear normal mode in a non-local sense. Thus, if one can find the exact solution of Equation (4), this solution will describe the exact shape of the manifold. However, solving Equation (4) is, in general, not possible.

For weakly nonlinear systems, an approximate local solution can be computed by assuming a polynomial expansion of $X_{i}$ and $Y_{i}$ with respect to $u_{k}$ and $v_{k}$ up to the desired order as

$$
\left\{\begin{aligned}
X_{i}= & a_{1, i}^{k} u_{k}+a_{2, i}^{k} v_{k}+a_{3, i}^{k} u_{k}^{2}+a_{4, i}^{k} u_{k} v_{k}+a_{5, i}^{k} v_{k}^{2} \\
& +a_{6, i}^{k} u_{k}^{3}+a_{7, i}^{k} u_{k}^{2} v_{k}+a_{8, i}^{k} u_{k} v_{k}^{2}+a_{9, i}^{k} v_{k}^{3}+\cdots, \\
Y_{i}= & b_{1, i}^{k} u_{k}+b_{2, i}^{k} v_{k}+b_{3, i}^{k} u_{k}^{2}+b_{4, i}^{k} u_{k} v_{k}+b_{5, i}^{k} v_{k}^{2} \\
& +b_{6, i}^{k} u_{k}^{3}+b_{7, i}^{k} u_{k}^{2} v_{k}+b_{8, i}^{k} u_{k} v_{k}^{2}+b_{9, i}^{k} v_{k}^{3}+\cdots
\end{aligned}\right.
$$

Substituting Equation (5) into Equation (4) and equating coefficients of like powers in $u_{k}$ and $v_{k}$ yields a set of linear equations which can be solved, one order at a time, for the $a_{j, i}^{k}$ 's and $b_{j, i}^{k}$ 's. These coefficients govern the nonlinear contributions (at various orders) of the $i$ th linear mode to the $k$ th nonlinear mode.

The reduced differential equation, which governs motions on the manifold, is determined by back substitution of the $X_{i}$ 's and $Y_{i}$ 's into Equation (1) for $i=k$. The dynamics of $u_{k}(t)$ may then be determined (for example by numerical integration), and the remaining coordinates obtained through the relations in Equation (5). This process requires solving only one nonlinear ordinary differential equation (ODE) to determine the motion on each nonlinear normal mode, as compared to $N$ coupled ODE's involved in a direct integration of Equation (1). It can be shown that, for systems where the lowest nonlinearity is of order $Q$, the dynamics are precise through order $\left(N^{\prime}+Q-1\right)$ where $N^{\prime}$ is the order of approximation of the manifold. For general nonlinearities, this means that the approximation of the dynamics is precise to one order higher than that of the manifold itself, and two orders higher in the case of purely odd nonlinearities. However, additional higher order terms, while not precise, may yield more accurate equations of motion under certain conditions. 


\subsection{Specialized Single-Mode Solution}

In order to develop approximate solutions for Equation (4) which may be applied to a wide variety of nonlinear structural systems, yet remain somewhat tractable, several assumptions are necessary. In this formulation, the following assumptions are made:

- The equations are decoupled to linear order using $N$ linear system modes, and thus expressed in terms of the linear normal coordinates. This form may be easily obtained for large structural systems, for example using modal analysis in conjunction with a finite element representation.

- Damping is not considered. However, small damping may be subsequently added to the reduced equation set.

- The nonlinearities are of second and third order only in displacement.

- The manifold is single-valued and analytic for the amplitudes under consideration.

These assumptions allow the equations of motion to be written in the following, second-order form:

$$
\ddot{\mathbf{x}}+[\Omega] \mathbf{x}+\mathbf{f}^{\mathrm{nl}}(\mathbf{x})=0,
$$

where

$$
[\Omega]=\left[\begin{array}{ccc}
\omega_{1}^{2} & 0 & 0 \\
0 & \ddots & 0 \\
0 & 0 & \omega_{N}^{2}
\end{array}\right]
$$

and $\omega_{i}$ is the $i$ th natural frequency of the linearized system. The nonlinear forcing, $\mathbf{f}^{\mathrm{nl}}$ may be expressed as:

$$
\mathbf{f}^{\mathbf{n l}}=[\alpha] \mathbf{x}^{2 *}+[\beta] \mathbf{x}^{3 *},
$$

where $\mathbf{x}^{2 *}$ contains all unique second-order combinations, and $\mathbf{x}^{3 *}$ contains all unique thirdorder combinations within the vector $\mathbf{x}$. Consequently, the non-square matrices $[\alpha]$ and $[\beta]$ contain the coefficients for every possible quadratic or cubic coupling in the (modal) displacement $\mathbf{x}$. The individual elements in $[\alpha]$ and $[\beta]$ are denoted as: $\alpha_{j, l}^{i}, \beta_{j, l, m}^{i}$, where $i$ indicates the row in $[\alpha]$ or $[\beta]$; and $j, l, m$ indicate the coupled degrees of freedom. In this notation, the order of the subscripts is extraneous, that is, $\alpha_{1,2}^{3}=\alpha_{2,1}^{3}$, and both refer to the same element in $[\alpha]$. Note that in order to obtain these elements, one must transform the nonlinear terms from physical to modal coordinates, a somewhat tedious procedure.

Now, $f_{i}$ from Equation (4) may be written as:

$$
f_{i}=-\omega_{i}^{2} X_{i}-\sum_{j=1}^{N} \sum_{l=j}^{N} \alpha_{j, l}^{i} X_{j} X_{l}-\sum_{j=1}^{N} \sum_{l=j}^{N} \sum_{m=l}^{N} \beta_{j, l, m}^{i} X_{j} X_{l} X_{m} .
$$

We seek a local solution for the $X_{i}$ 's and $Y_{i}$ 's in terms of $u_{k}$ and $v_{k}$ in the form of the expansion above, Equation (5). 
Due to the choice of modal coordinates, the first-order solution is trivial:

$$
\begin{aligned}
& a_{1, i}^{k}=\delta_{i k}, \\
& a_{2, i}^{k}=0, \\
& b_{1, i}^{k}=0, \\
& b_{2, i}^{k}=\delta_{i k} .
\end{aligned}
$$

Here, $\delta_{i k}$ is the Kronecker delta. The higher-order coefficients may be determined by substituting Equations (7) and (5) into Equation (4) and collecting like powers in $u_{k}$ and $v_{k}$ to produce a linear set of equations, a task greatly simplified by symbolic processors such as Mathematica $^{\mathrm{TM}}$. Using this formulation, the second-order coefficients are:

$$
\begin{aligned}
& a_{3, i}^{k}=\frac{\alpha_{k, k}^{i}\left(2 \omega_{k}^{2}-\omega_{i}^{2}\right)}{\omega_{i}^{2}\left(\omega_{i}^{2}-4 \omega_{k}^{2}\right)}, \\
& a_{5, i}^{k}=\frac{2 \alpha_{k, k}^{i}}{\omega_{i}^{2}\left(\omega_{i}^{2}-4 \omega_{k}^{2}\right)}, \\
& b_{4, i}^{k}=\frac{-2 \alpha_{k, k}^{i}}{\omega_{i}^{2}-4 \omega_{k}^{2}} .
\end{aligned}
$$

In addition, $a_{4, i}^{k}=b_{3, i}^{k}=b_{5, i}^{k}=0$. At third order, $a_{7, i}^{k}=a_{9, i}^{k}=b_{6, i}^{k}=b_{8, i}^{k}=0$, and,

$$
\begin{aligned}
a_{6, i}^{k} & =\frac{-C_{1, i}^{1, k}-\beta_{k, k, k}^{i}+\alpha_{k, k}^{k} b_{4, i}^{k}}{\omega_{i}^{2}}-\frac{\omega_{k}^{2}\left[C_{2, i}^{1, k}-C_{3, i}^{1, k}\left(3 \omega_{k}^{2}-\omega_{i}^{2}\right)\right]}{\omega_{i}^{2} D_{i}^{1, k}}, \\
a_{8, i}^{k} & =\frac{C_{3, i}^{1, k}}{2 \omega_{i}^{2} \omega_{k}^{2}}-\frac{\left(3 \omega_{k}^{2}-\omega_{i}^{2}\right)\left[C_{2, i}^{1, k}-C_{3, i}^{1, k}\left(3 \omega_{k}^{2}-\omega_{i}^{2}\right)\right]}{2 \omega_{i}^{2} \omega_{k}^{2} D_{i}^{1, k}}, \\
b_{7, i}^{k} & =\frac{-\left[C_{2, i}^{1, k}-C_{3, i}^{1, k}\left(3 \omega_{k}^{2}-\omega_{i}^{2}\right)\right]}{D_{i}^{1, k}} \\
b_{9, i}^{k} & =a_{8, i}^{k} .
\end{aligned}
$$

The constants $C_{p, i}^{1, k}$, and $D_{i}^{1, k}$ are defined as:

$$
\begin{aligned}
C_{1, i}^{1, k} & =\sum_{m=1}^{N} a_{3, m}^{k} \alpha_{k, m}^{i}, \\
C_{2, i}^{1, k} & =2 \omega_{i}^{2} \omega_{k}^{2} \sum_{m=1}^{N} a_{5, m}^{k} \alpha_{k, m}^{i}, \\
C_{3, i}^{1, k} & =-3\left(C_{1, i}^{1, k}+\beta_{k, k, k}^{i}-\alpha_{k, k}^{k} b_{4, i}^{k}\right)-2 \omega_{i}^{2} \alpha_{k, k}^{k} a_{5, i}^{k}, \\
D_{i}^{1, k} & =\left(\omega_{k}^{2}-\omega_{i}^{2}\right)\left(\omega_{i}^{2}-9 \omega_{k}^{2}\right) .
\end{aligned}
$$

Note that the third-order coefficients depend on the second-order results, as is typical in perturbation expansions. Similar results have been found for fourth- and fifth-order coefficients, but the general analytic results are omitted for the sake of brevity. 


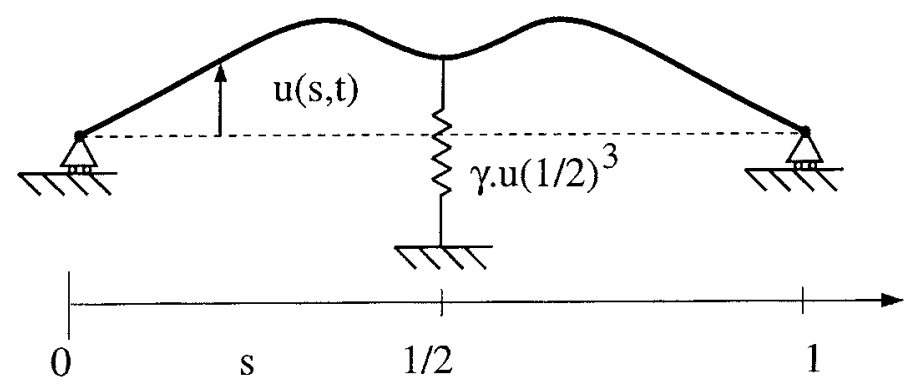

Figure 1. Simply-supported Euler-Bernoulli (linear) beam connected to a purely cubic spring.

Once the above expansion coefficients are calculated, all degrees of freedom are known, albeit approximate, functions of $u_{k}$ and $v_{k}$. These expressions may be substituted into the $k$ th equation of motion to produce a single, second-order, nonlinear oscillator in $u_{k}$ and $v_{k}$. This oscillator governs the local dynamics on the $k$ th invariant manifold.

\subsection{Analytic Example: A Simply Supported Euler-Bernoulli Beam CONSTRAINED BY A NONLINEAR SPRING}

The above methodology is applied to a homogeneous, uniform, simply supported EulerBernoulli beam with a nonlinear cubic spring attached at the center - see Figure 1. While the beam itself is assumed to deform in the linear range, the spring is chosen as purely cubic so that the linearized system's normal modes are those of the simply supported beam alone (i.e., pure sine waves). With this choice, the influence of the various linear modes on the nonlinear ones can be visualized easily. Notice that since the spring is located at a node of the antisymmetric (even numbered) modes, they remain unaffected (linear). Therefore, only the symmetric (odd numbered) normal modes are influenced by the nonlinear spring and, furthermore, they feature only contributions from the other symmetric linear modes.

The beam has the following equation for transverse motion, in non-dimensional form:

$$
\left.\ddot{u}+\lambda u_{, s s s s}+\mu u^{3} \delta\left(s-\frac{1}{2}\right)=0, \quad s \in\right] 0,1[,
$$

where $\lambda=E I / m, \mu=\gamma / m, E$ is Young's modulus, $I$ is the second moment of area of a cross section, $m$ is the mass per unit length, $\gamma$ is the nonlinear stiffness of the spring, $s$ represents the spatial coordinate along the beam, $u(s, t)$ is the transverse deflection of the beam, $(\cdot)_{s}$ denotes a derivative with respect to $s$, an overdot represents a derivative with respect to time, and $\delta(\cdot)$ is the Dirac function. The associated boundary conditions are $u(0)=u(1)=0$, and $u_{, s s}(0)=u_{, s s}(1)=0$. For this example, we take $\lambda=1$, and $\mu=10^{4}$ when numerical results are shown.

The beam deflection, $u(s, t)$, is first discretized using the natural modes of the linearized system, $\phi_{j}(s)=\sin (j \pi s)$,

$$
u(s, t) \simeq \sum_{j=1}^{N} x_{j}(t) \phi_{j}(s),
$$


where $N$ is the number of terms in the expansion, i.e., the number of modes that would be retained for a linear modal analysis of the nonlinear system. Projection of the equation of motion onto the $i$ th linear mode yields

$$
\ddot{x}_{i}+\lambda(i \pi)^{4} x_{i}+2 \mu\left[\sum_{j=1}^{N} x_{j} \sin \left(j \frac{\pi}{2}\right)\right]^{3} \sin \left(i \frac{\pi}{2}\right)=0, \quad i=1, \ldots, N,
$$

which can be written in first-order form as

$$
\left\{\begin{array}{l}
\dot{x}_{i}=y_{i}, \\
\dot{y}_{i}=f_{i}\left(x_{1}, \ldots, x_{N}, y_{1}, \ldots, y_{N}\right),
\end{array} \quad i=1, \ldots, N,\right.
$$

where the inter-modal coupling effects are captured by

$$
f_{i}=-\lambda(i \pi)^{4} x_{i}-2 \mu\left[\sum_{j=1}^{N} x_{j} \sin \left(j \frac{\pi}{2}\right)\right]^{3} \sin \left(i \frac{\pi}{2}\right) .
$$

The set of differential equations, Equation (15), is what is simulated for a typical linear modal analysis. Alternatively, the procedure described earlier in this section can be applied to Equation (15) to determine the third- or higher-order approximation of the nonlinear normal modes of the system. The $k$ th nonlinear mode is given by, to third order [10]:

$$
x_{k}=u_{k}, \quad \dot{x}_{k}=v_{k}
$$

and for $i=1, \ldots, N, i \neq k$ :

$$
\begin{cases}x_{i}=\dot{x}_{i}=0, & \\ x_{i}=a_{6, i}^{k} u_{k}^{3}+a_{8, i}^{k} u_{k} v_{k}^{2}+\cdots, & (i \text { oden }) \\ \dot{x}_{i}=b_{7, i}^{k} u_{k}^{2} v_{k}+b_{9, i}^{k} v_{k}^{3}+\cdots, & (i \text { odd })\end{cases}
$$

where, if $k$ is even, $a_{6, i}^{k}=a_{8, i}^{k}=0$, and if $k$ is odd, for $i=1, \ldots, N, i \neq k$,

$$
\left\{\begin{array}{l}
a_{6, i}^{k}=2 \mu(-1)^{(k+i) / 2} \frac{\left(i^{4}-7 k^{4}\right)}{\lambda \pi^{4}\left(i^{4}-k^{4}\right)\left(i^{4}-9 k^{4}\right)}, \\
a_{8, i}^{k}=-12 \mu(-1)^{(k+i) / 2} \frac{1}{\lambda^{2} \pi^{8}\left(i^{4}-k^{4}\right)\left(i^{4}-9 k^{4}\right)},
\end{array}\right.
$$

and, for all $k$ with $i=1, \ldots, N, i \neq k, i$ odd:

$$
\left\{\begin{array}{l}
b_{7, i}^{k}=-2 \lambda(\pi k)^{4} a_{8, i}^{k}+3 a_{6, i}^{k} \\
b_{9, i}^{k}=a_{8, i}^{k}
\end{array}\right.
$$

Though different in form, equivalent expressions may be obtained using Equations (9)-(11).

Next, the deflection and velocity of the beam in the $k$ th nonlinear mode, $u^{k}(s, t)$ and $v^{k}(s, t)$, can be expressed in terms of the $k$ th nonlinear modal coordinate, $u_{k}(t)$, and the associated modal velocity, $v_{k}(t)$, as

$$
u^{k}(s, t)=u_{k} \sin (k \pi s)+\sum_{\substack{i \text { odd } \\ i \neq k}}\left[a_{6, i}^{k} u_{k}^{3}+a_{8, i}^{k} u_{k} v_{k}^{2}\right] \sin (i \pi s)+\cdots
$$




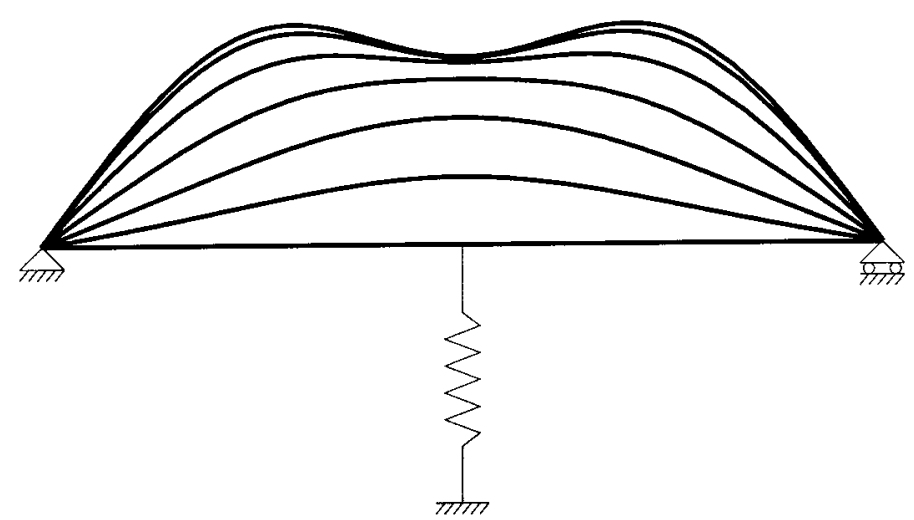

Figure 2. Beam displacement through a quarter-period of motion in the first nonlinear normal mode, sampled at seven equal time intervals. $u_{1}(0)=0, v_{1}(0)=6.93$.

$$
v^{k}(s, t)=v_{k} \sin (k \pi s)+\sum_{\substack{i \text { odd } \\ i \neq k}}\left[b_{7, i}^{k} u_{k}^{2} v_{k}+b_{9, i}^{k} v_{k}^{3}\right] \sin (i \pi s)+\cdots .
$$

Note that the nonlinear normal modes indicate synchronous motions for this system. That is, when $v_{k}=0$, the entire velocity field, $v^{k}(s, t)$, must also vanish. Similarly, since the nonlinearities are odd (that is, symmetric about the equilibrium), all points along the beam pass through zero simultaneously - specifically, when $u_{k}=0, u^{k}(s, t)=0$ over the entire beam. (For an example of a synchronous motion with even-order nonlinearities present, in which case the latter observation does not hold, see [8].) Here, $u^{k}(s, t)$ is the deflection of the point located at coordinate $s$ at time $t$ when the system undergoes a motion in the $k$ th nonlinear normal mode. It should not to be confused with $u_{k}(t)$, which is the nonlinear modal coordinate and is not meant to represent the motion of any particular point.

The dynamics of the nonlinear modes are governed by

$$
\begin{aligned}
\ddot{u}_{k} & +\lambda(k \pi)^{4} u_{k}+2 \mu \sin ^{2}(k \pi / 2) u_{k}^{3} \\
& +6 \mu u_{k}^{3}\left(\sum_{\substack{i \text { odd } \\
i \neq k}}\left[a_{6, i}^{k} u_{k}^{2}+a_{8, i}^{k} v_{k}^{2}\right] \sin (i \pi / 2)\right) \sin (k \pi / 2)=0,
\end{aligned}
$$

for $k=1, \ldots, N$. Note that the dynamics of the $N$ nonlinear modal oscillators are individually decoupled from one another, and that a third-order manifold accurately produces these equations of motion through fifth order.

Figure 2 shows the position of the beam at seven equidistant instants of time for a motion purely in the first nonlinear mode, as generated by Equations (19-21). Note that as the beam undergoes its oscillation, the shape does not remain constant, and that the spring influence increases with displacement amplitude. These geometric effects, and their mathematical repercussions cannot be captured through a single linear mode truncation of the system. Simply adding linear modes does increase model accuracy. However, without the constraints provided by the manifold, the response loses periodicity.

Figures 3 and 4 display simulation results obtained using this procedure, along with results obtained with classical linear modal analyses of the nonlinear system performed with various 


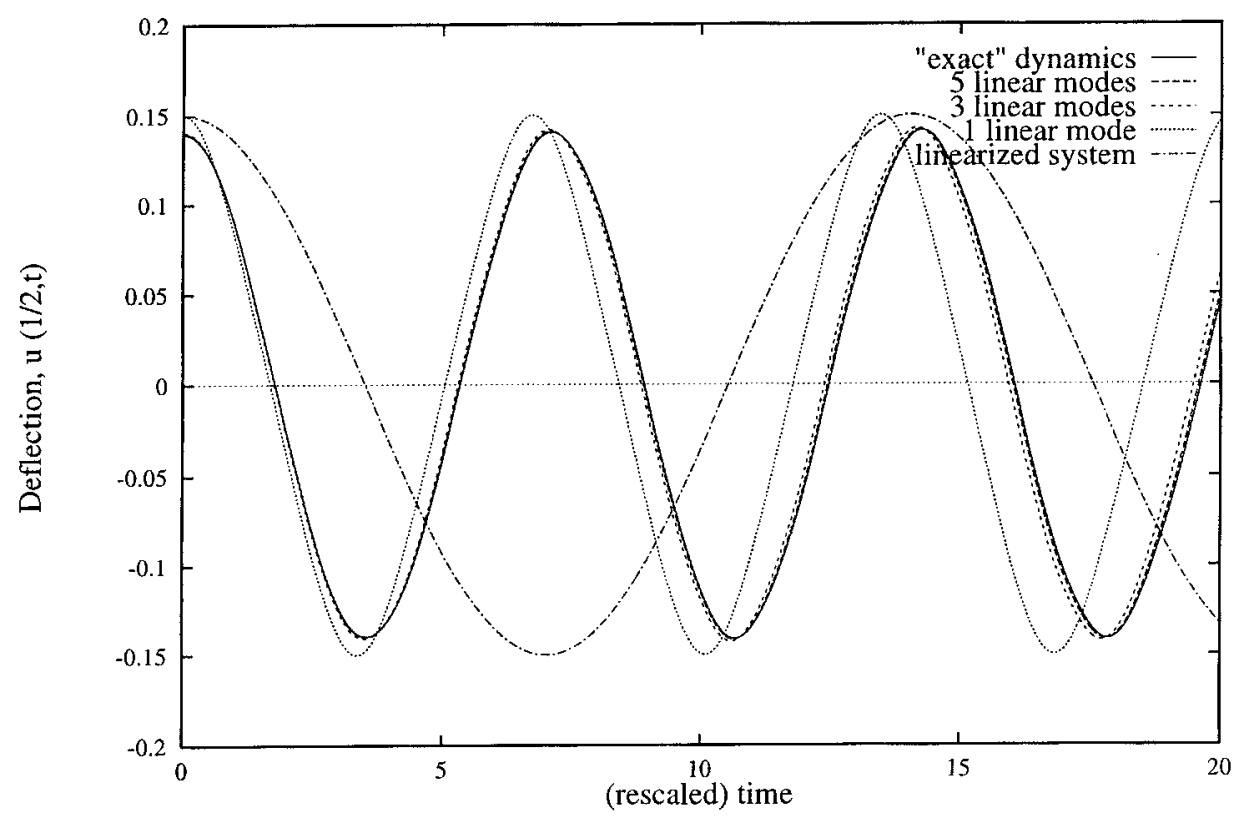

Figure 3. Deflection of the middle-point of the beam obtained by various linear modal analysis simulations initiated on the first nonlinear normal mode manifold. Initial conditions: $u_{1}(0)=0.15, v_{1}(0)=0$.

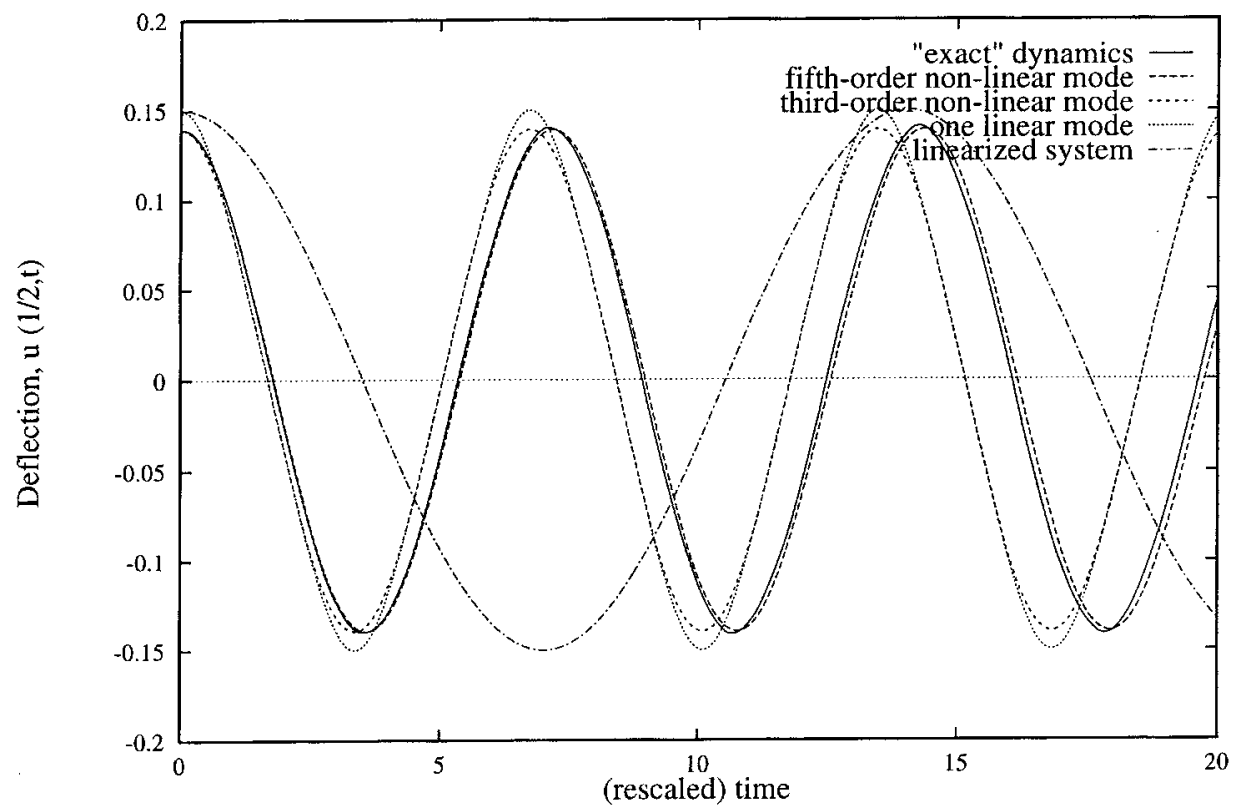

Figure 4. Deflection of the middle-point of the beam obtained by various simulations initiated on the first nonlinear normal mode manifold. Initial conditions: $u_{1}(0)=0.15, v_{1}(0)=0$. 
numbers of modeled linear modes. In both figures, the 'exact' solution was determined using a linear modal analysis with 25 linear modes; that is, by simulating the 25-degrees-of-freedom system obtained by projecting the equations of motion onto the lower 25 linear modes, using initial conditions which lie on the third-order approximation of the first nonlinear normal mode manifold in the 50-dimensional phase space. In this particular case it is seen that at least three to five linear modes are necessary to achieve an accuracy comparable to that obtained with a single fifth-order nonlinear mode as obtained above. The nonlinear mode results are obtained by simulation of a single second-order differential equation (Equation (21)), which includes the influence of the other linear modes. In the case where one uses a single linear mode through the entire analysis, the influence of the other linear modes is completely missing, whereas it is naturally embedded in the nonlinear normal mode. See Figure 4 which compares several simulations, all utilizing only one ODE, to the 'exact' solution. It should be noted that the initial displacements shown in these figures include contributions from chosen mode $\left(u_{1}\right)$, as well as the non-modeled modes. Consequently, the initial displacement will vary as additional modes are included, i.e., as the manifold is projected into a larger configuration space.

\section{Multi-Mode Invariant Manifolds}

The potential of nonlinear normal modes is evident from the previous section. However, it is important to note that, by definition, they are only individually invariant. Therefore, though they do not interact when the system undergoes a motion in any one of the modal manifolds, nothing prevents them from interacting during an arbitrary motion. This immediately reminds one of the contamination problems encountered during the linear modal analysis of nonlinear systems - an issue which was precisely at the origin of the definition of individual nonlinear normal modes.

In order to properly remove the contamination due to nonlinear modes which are not included in the reduced-order model, a new, more general, formulation is necessary, such that the individually invariant nonlinear normal modes are but a special case. The underlying idea is to generate high-dimensional invariant manifolds, referred to as multi-mode manifolds, essentially in the same manner as the individually invariant manifolds were produced in Section 2. These multi-mode manifolds, when comprising the influence of $M$ nonlinear modes, are of dimension $2 M$ in the phase space for the oscillatory systems typically of interest in structural dynamics. These multi-mode manifolds are still not completely invariant, as two different multi-mode manifolds would interact during a general motion, just as the individual nonlinear normal modes did. However, for motions on a given multi-mode manifold, invariance is ensured between itself and the rest of the (non-modeled) nonlinear modes.

Consequently, for a system for which $M$ nonlinear modes are to be modeled and for which the remaining ones are to be merely ignored, the multi-mode manifold should comprise all $M$ modes, so that (1) the interactions between those $M$ modes can be accounted for, and (2) the interactions with the non-modeled modes can be completely removed. If a mode is non-modeled despite an internal resonance with a modeled one, the mathematical process of generating the multi-mode manifold will become singular, thereby detecting the anomaly.

The procedure to determine multi-mode invariant manifolds follows closely the one presented in Section 2. If $S_{M}$ denotes the subset of indices corresponding to the modeled modes, and $\mathbf{u}_{M}$ and $\mathbf{v}_{M}$ represent the vectors of the corresponding nonlinear modal coordinates and velocities, then the various linear modal coordinates are expressed as functions of the modeled 
modes as

$$
\begin{aligned}
& \left\{\begin{array}{l}
x_{k}=u_{k}, \\
y_{k}=v_{k}, \quad \text { for } \quad k \in S_{M}
\end{array}\right. \\
& \left\{\begin{array}{l}
x_{j}=X_{j}\left(\mathbf{u}_{M}, \mathbf{v}_{M}\right), \\
y_{j}=Y_{j}\left(\mathbf{u}_{M}, \mathbf{v}_{M}\right) .
\end{array} \text { for } j \notin S_{M},\right.
\end{aligned}
$$

Taking the time-derivatives of Equation (23) yields:

$$
\begin{cases}Y_{j}=\dot{X}_{j}=\sum_{k \in S_{M}}\left(\frac{\partial X_{j}}{\partial u_{k}} v_{k}+\frac{\partial X_{j}}{\partial v_{k}} f_{k}\right), & \text { for } j \notin S_{M}, \\ f_{j}=\dot{Y}_{j}=\sum_{k \in S_{M}}\left(\frac{\partial Y_{j}}{\partial u_{k}} v_{k}+\frac{\partial Y_{j}}{\partial v_{k}} f_{k}\right) . & \end{cases}
$$

As before, exact analytical solutions to these equations are not readily available, and in most cases (namely, for weakly nonlinear systems) approximations will be sought in a series expansion form. One has, to third order:

$$
\begin{aligned}
X_{j}\left(\mathbf{u}_{M}, \mathbf{v}_{M}\right)= & \sum_{k \in S_{M}}\left(a_{1, j}^{k} u_{k}+a_{2, j}^{k} v_{k}\right)+\sum_{k \in S_{M}} \sum_{l \in S_{M}}\left(a_{3, j}^{k, l} u_{k} u_{l}+a_{4, j}^{k, l} u_{k} v_{l}+a_{5, j}^{k, l} v_{k} v_{l}\right) \\
& +\sum_{k \in S_{M}} \sum_{l \in S_{M}} \sum_{q \in S_{M}}\left(a_{6, j}^{k, l, q} u_{k} u_{l} u_{q}+a_{7, j}^{k, l, q} u_{k} u_{l} v_{q}\right. \\
& \left.+a_{8, j}^{k, l, q} u_{k} v_{l} v_{q}+a_{9, j}^{k, l, q} v_{k} v_{l} v_{q}\right)+\cdots, \\
Y_{j}\left(\mathbf{u}_{M}, \mathbf{v}_{M}\right)= & \sum_{k \in S_{M}}\left(b_{1, j}^{k} u_{k}+b_{2, j}^{k} v_{k}\right)+\sum_{k \in S_{M}} \sum_{l \in S_{M}}\left(b_{3, j}^{k, l} u_{k} u_{l}+b_{4, j}^{k, l} u_{k} v_{l}+b_{5, j}^{k, l} v_{k} v_{l}\right) \\
& +\sum_{k \in S_{M}} \sum_{l \in S_{M}} \sum_{q \in S_{M}}\left(b_{6, j}^{k, l, q} u_{k} u_{l} u_{q}+b_{7, j}^{k, l, q} u_{k} u_{l} v_{q}\right. \\
& \left.+b_{8, j}^{k, l, q} u_{k} v_{l} v_{q}+b_{9, j}^{k, l, q} v_{k} v_{l} v_{q}\right)+\cdots .
\end{aligned}
$$

Note that this decomposition is not unique, and that the number of coefficients of order $p$ when $M$ nonlinear modes are modeled is, for each $X_{j}$ and $Y_{j}$ :

$$
C_{p}^{2 M-1+P}=\frac{(2 M-1+p) !}{(2 M-1) ! p !}
$$

which increases very rapidly with both $p$ and $M$. Substituting Equations (25) and (26) into the $j$ th pair of manifold equations, Equation (24), and equating like powers in $\mathbf{u}_{m}$ and $\mathbf{v}_{m}$, one obtains the coefficients, one order at a time. 


\subsection{Specialized Multi-Mode Solution}

As with the single-mode case, it is necessary to restrict the eligible systems in order that the solutions remain marginally tractable. Consequently, the same restrictions as those introduced in Section 2.1 are applied. In addition, in order to ensure a unique solution, it is assumed that the non-unique coefficients of Equations (25) and (26) are equal. That is, because

$$
u_{k} u_{l} v_{q}=u_{l} u_{k} v_{q} \neq u_{k} u_{q} v_{l}
$$

we enforce

$$
a_{7, j}^{k, l, q}=a_{7, j}^{l, k, q},
$$

but allow

$$
a_{7, j}^{k, l, q} \neq a_{7, j}^{k, q, l} .
$$

Also, as in the single mode case, these are local results, and their fidelity depends on the amplitude of motion as well as the proximity to internal resonances between modeled and non-modeled modes.

Although each order may be solved sequentially in its entirety, resulting in large matrix equations for each complete set of second- and third-order coefficients, the process may be simplified by dividing it into several subproblems according to the number of distinct modes contained in the coefficient superscripts. That is, one first solves for terms like $a_{6, i}^{k, k, k}$, then for terms like $a_{6, i}^{k, k, l}$, where $k \neq l$, and finally for those like $a_{6, i}^{k, l, q}$ where $k, l$ and $q$ are all distinct. These are referred to as the one-mode, two-mode, and three-mode coefficients, respectively. The one-mode problem is almost identical to that of the single mode manifold, and the same solutions may be used. However, the numerical answers are not identical, as the inclusion of more modes eliminates some of the expansion coefficients (e.g., $a_{3, j}^{k, k}=0$ if $j \in S_{m}$ ), and the values of the constants $C_{p, i}^{1, k}$, as defined in Equation (11), are consequently altered. That is, certain constraints are eliminated when an additional mode is included in $S_{m}$, and this change is reflected in the reduced equations of motion.

The two-mode problem may be solved first for the second-order expansion coefficients, and then for the appropriate third-order coefficients. The problems are both linear, but due to the size and complexity of the explicit solutions, they are presented here in matrix form only. In addition, the coefficients which are zero have been eliminated from the matrix formulation. It is found that in the one-, two- and three-mode problems the expansion coefficients $a_{4}, a_{7}, a_{9}, b_{3}, b_{5}, b_{6}, b_{8}$ are all zero. This is due to the fact that we are considering conservative, non-gyroscopic systems, which eliminates the possibility of such terms appearing. For the two-mode problem, the second-order coefficients may be determined by solving

$$
\left[\begin{array}{cccc}
-2 & 2 \omega_{k}^{2} & 1 & 0 \\
-2 & 2 \omega_{l}^{2} & 0 & 1 \\
-2 \omega_{i}^{2} & 0 & \omega_{l}^{2} & \omega_{k}^{2} \\
0 & 2 \omega_{i}^{2} & 1 & 1
\end{array}\right]\left[\begin{array}{c}
a_{3, i}^{k, l} \\
a_{5, i}^{k, l} \\
b_{4, i}^{k, l} \\
b_{4, i}^{l, k}
\end{array}\right]=\left[\begin{array}{c}
0 \\
0 \\
\alpha_{k, l}^{i} \\
0
\end{array}\right]
$$

while the third-order coefficients are obtained by solving 


$$
\left[\begin{array}{cccccc}
-3 & 2 \omega_{k}^{2} & 0 & 1 & 0 & 0 \\
-6 & 2 \omega_{l}^{2} & 2 \omega_{k}^{2} & 0 & 2 & 0 \\
0 & -2 & -1 & 0 & 0 & 3 \\
-3 \omega_{i}^{2} & 0 & 0 & \omega_{l}^{2} & 2 \omega_{k}^{2} & 0 \\
0 & -2 \omega_{i}^{2} & 0 & -2 & -2 & 6 \omega_{k}^{2} \\
0 & 0 & -\omega_{i}^{2} & 0 & -2 & 3 \omega_{l}^{2}
\end{array}\right]\left[\begin{array}{c}
a_{6, i}^{k, k, l} \\
a_{8, i}^{k, k, l} \\
a_{8, i}^{l, k, k} \\
b_{7, i}^{k, k, l} \\
b_{7, i}^{l, k, k} \\
b_{9, i}^{k, k, l}
\end{array}\right]=\left[\begin{array}{c}
-C_{1, i}^{3, k, l} \\
-C_{2, i}^{3, k, l} \\
0 \\
C_{7, i}^{3, k, l} \\
C_{5, i}^{3, k, l} \\
C_{6, i}^{3, k, l}
\end{array}\right]
$$

For the three-mode problem, the third-order coefficients are the solution of:

$$
\left[\begin{array}{cccccccc}
-6 & 2 \omega_{k}^{2} & 2 \omega_{l}^{2} & 0 & 2 & 0 & 0 & 0 \\
-6 & 0 & 2 \omega_{m}^{2} & 2 \omega_{k}^{2} & 0 & 2 & 0 & 0 \\
-6 & 2 \omega_{m}^{2} & 0 & 2 \omega_{l}^{2} & 0 & 0 & 2 & 0 \\
0 & -1 & -1 & -1 & 0 & 0 & 0 & 3 \\
-3 \omega_{i}^{2} & 0 & 0 & 0 & \omega_{m}^{2} & \omega_{l}^{2} & \omega_{k}^{2} & 0 \\
0 & -2 \omega_{i}^{2} & 0 & 0 & -2 & 0 & -2 & 6 \omega_{l}^{2} \\
0 & 0 & -2 \omega_{i}^{2} & 0 & -2 & -2 & 0 & 6 \omega_{k}^{2} \\
0 & 0 & 0 & -2 \omega_{i}^{2} & 0 & -2 & -2 & 6 \omega_{m}^{2}
\end{array}\right]\left[\begin{array}{c}
a_{6, i}^{k, l, m} \\
a_{8, i}^{l, m, k} \\
a_{8, i}^{k, l, m} \\
a_{8, i}^{m, l, k} \\
b_{7, i}^{k, l, m} \\
b_{7, i}^{k, m, l} \\
b_{7, i}^{,, m, k} \\
b_{9, i}^{k, l, m}
\end{array}\right]=\left[\begin{array}{c}
C_{8, i}^{3, k, l, m} \\
C_{8, i}^{3, k, m, l} \\
C_{8, i}^{3, l, m, k} \\
0 \\
C_{9, i}^{3, k, l, m} \\
C_{10, i}^{3, l, m} \\
C_{10, i}^{3, k, l, m} \\
C_{10, i}^{3, m}, l, k
\end{array}\right]
$$

In Equations (27-29), each equation depends on a specific $i \notin S_{M}$ and $k, l, m \in S_{M}$. The constants $C_{p, i}^{3, k, l}$ depend on the first- and second-order results, and are shown explicitly in the appendix. These matrix expressions were initially formulated by hand, and then checked using the symbolic processor Mathematica ${ }^{T M}$. Due to the relatively sparse nature of these matrices, they may be symbolically inverted to yield analytic expressions for the expansion coefficients which depend only on $[\alpha],[\beta]$, the $\omega$ 's and the lower-order expansion coefficients. Though these expressions are rather large and unwieldy, they may easily be implemented in a computer code and used to calculate systematically all of the expansion coefficients. It should be noted that the symbolic approach used here allows $k, l, m$ in Equations (27-29) to represent any three modeled modes, and the corresponding (symbolic) solutions will be valid for any three modeled modes. Alternatively, numerical values may be used in Equations (27-29), and the inversion may be repeated for each combination of modeled modes. If several modes are chosen, this may entail considerable work each time a solution is sought. The symbolic approach avoids this shortcoming by producing a single, explicit, general purpose solution. However, due to its complexity, this approach may no longer be practical if additional nonlinear terms or expansion coefficients are included in the formulation (for example, those arising from gyroscopic terms). However, even if additional effects are added, making symbolic inversion impractical, the sub-problems above are still more desirable than the explicit, simultaneous solution of all coefficients at a given order.

While internal resonances between modeled modes are automatically accounted for, internal resonances between modeled and non-modeled modes will cause singularities in the analytic solutions. There are many possible internal resonances. Through third order, they are:

$$
\begin{array}{ll}
\omega_{i}=\omega_{k}, & \omega_{i}=3 \omega_{k}, \\
\omega_{i}=2 \omega_{k}, & \omega_{i}=\left|2 \omega_{k} \pm \omega_{l}\right|, \\
\omega_{i}=\left|\omega_{k} \pm \omega_{l}\right|, & \omega_{i}=\left|\omega_{k} \pm \omega_{l} \pm \omega_{m}\right|,
\end{array}
$$


where $(i, k, l, m) \in S_{M}$.

Once the expansion coefficients are known, the non-modeled degrees of freedom can be expressed as approximate functions of the modeled coordinates. These functions may be substituted back into the equations of motion corresponding to the modeled modes, thus eliminating the non-modeled modes from the original set of second-order differential equations. This reduction process may be automated, producing a set of equations with polynomial nonlinearities in the modeled coordinates. As in the single-mode case, a third-order expansion of the non-modeled coordinates produces equations which are precise through fourth order if $[\alpha]$ is non-zero, and fifth order if $[\alpha]=0$. In addition, it has been found that, even when $[\alpha]$ is non-zero, model fidelity is considerably improved when the fifth-order terms are retained. Empirical evidence suggests that, while not precise, these additional terms contain essential information.

As alluded to above, a computer program has been developed for the multi-mode case which - given $[\alpha],[\beta]$, and the system's linear natural frequencies - can reduce a system of $N$ equations which are formulated in the linear modal coordinates, down to a system of $M$ equations of motion, in $M$ chosen nonlinear modal coordinates i.e., a reduced-order model. This program generates the complete, third-order, $M$-mode manifold via the analytic solutions to Equations (27-29), enabling the $M$ equations of motion to be expressed to fifth order. This means it is now feasible to systematically (and automatically) reduce many nonlinear structural systems to include only the modes of interest without eliminating all effects due to non-modeled modes. For example, a two mode manifold may be produced to examine an internal resonance between two modes, yielding equations which not only account for all interactions between the two chosen modes, but include relevant effects due to all other modes. Of course, these additional effects are embedded in the fourth- and fifth-order terms, of which there may be a great many. Consequently, some examination is necessary to determine when 'reducing' $N$ third-order equations to $M$ fifth-order equations will result in significant time savings; this matter is considered next.

\subsection{Some Computational Considerations}

Depending on the ratio of $M / N$, the simplification obtained from the nonlinear mode approach may be significant. One indication of the complexity of a given system is the total number of nonlinear terms in the model. For example, this is a measure of both the number of computations necessary at a given time step in a numerical simulation. In the original system, there are $N$ equations, with

$$
\frac{1}{6}\left(N^{3}+6 N^{2}+5 N\right)
$$

possible nonlinear terms in each equation (this and the following results are obtained by counting the terms at each order). By comparison, in the reduced system there are $M$ equations with

$$
\frac{M}{30}\left(4 M^{4}+30 M^{3}+85 M^{2}+105 M+46\right)
$$

terms each, when all generated fifth-order terms are retained (in order to be conservative). At the highest order, this difference reduces to an $M \times(4 / 5) M^{5}$ versus an $N \times N^{3}$ set of nonlinear terms. Consequently, there is not significant reduction in the number of terms if

$$
M \geq \approx N^{2 / 3}
$$


in the limiting case of the fifth-order result. It can be shown that for $N>4$, reduction will always occur if $M \leq \sqrt{N}$. This is not a distinct boundary, but it may be used as a general guide when applying this technique. These criteria are indicative of the most nonlinear cases. In simpler cases, when the $[\alpha]$ and $[\beta]$ matrices are sparse, the resulting reduced set may contain many terms which are also zero or of negligible magnitude. Due to these effects, an absolute description of the efficiency of this technique is not possible, and each case must be evaluated individually. For example, if $N=20$, and $[\alpha]$ and $[\beta]$ are full, there will be $20 \times 1750=35000$ original nonlinear terms. If this is reduced to $M=4$ equations, there will be, at most, $4 \times 636=2544$ nonlinear terms in the reduced set.

In many structural systems, it is primarily the low frequency modes which are of interest. However, when nonlinearities are present, it is often necessary to simulate the high frequency modes as well in order to achieve the desired accuracy in the modes of interest. This requires that the time steps of any integration scheme be small enough to reproduce the high frequency dynamics accurately. When these systems are reduced to their low frequency modes using a multi-mode manifold, the effects of the high frequency modes are captured in the reduced equations, and it is no longer necessary to simulate them explicitly, hence allowing for a time step governed by the low frequency modes. When this time savings is compounded with that due to the size reduction of the equations, the necessary computer time may be reduced by several orders of magnitude. In addition, it should be noted that the size of the reduced equations does not grow with $N$. Consequently, it is possible to produce models which are reduced from many more modes than would have previously been practical. Also, as $N$ is increased, the convergence of the reduced equations (with $M$ held constant) may be studied to guarantee their accuracy.

\subsection{Analytic Example: A Simply Supported Euler-Bernoulli BeAm CONSTRAINED BY A NONLINEAR SPRING}

In the particular case of the system depicted in Figure 1, a two-mode invariant manifold is computed with the aid of symbolic manipulation. Here, the first-order terms vanish except for those corresponding directly to the linear modes (since the linear modes are used to discretize the system), and all second-order terms are zero (since there are no quadratic nonlinearities). If $S_{m}=\{k, l\}$, the non-zero $a$ 's are as follows:

$$
\begin{aligned}
a_{6, j}^{k, k, k} & =\frac{2\left(-j^{4}+7 k^{4}\right) \mu \sin ^{3}(k \pi / 2) \sin (j \pi / 2)}{\lambda \pi^{4}\left(j^{8}-10 j^{4} k^{4}+9 k^{8}\right)}, \\
a_{6, j}^{k, k, l} & =6 \mu \sin ^{2}(k \pi / 2) \sin (l \pi / 2) \sin (j \pi / 2) \\
& \times\left(j^{8}-6 j^{4} l^{4}-2 j^{4} k^{4}-2 k^{4} l^{4}+l^{8}+8 k^{8}\right) /\left[\left(\lambda \pi^{4}\left(l^{4}-j^{4}\right)\right.\right. \\
& \left.\times\left(-8 k^{4} l^{4}-8 j^{4} k^{4}+16 k^{8}+j^{8}-2 j^{4} l^{4}+l^{8}\right)\right], \\
a_{8, j}^{k, k, k} & =\frac{12 \mu \sin ^{3}(k \pi / 2) \sin (j \pi / 2)}{\lambda^{2} \pi^{8}\left(j^{8}-10 j^{4} k^{4}+9 k^{8}\right)}, \\
a_{8, j}^{k, l, l} & =\frac{-12 \mu\left(3 k^{4}+j^{4}-4 l^{4}\right) \sin ^{2}(l \pi / 2) \sin (j \pi / 2) \sin (k \pi / 2)}{\lambda^{2} \pi^{8}\left(k^{4}-j^{4}\right)\left(-8 k^{4} l^{4}-8 j^{4} l^{4}+16 l^{8}+j^{8}-2 j^{4} k^{4}+k^{8}\right)},
\end{aligned}
$$




$$
a_{8, j}^{k, k, l}=\frac{24 \mu \sin ^{2}(k \pi / 2) \sin (l \pi / 2) \sin (j \pi / 2)}{\lambda^{2} \pi^{8}\left(-8 k^{4} l^{4}-8 j^{4} k^{4}+16 k^{8}+j^{8}-2 j^{4} l^{4}+l^{8}\right)},
$$

with similar relations when $k$ and $l$ are switched, from which one obtains, $b_{6, j}^{p, q, r}=b_{8, j}^{p, q, r}=0$ for all $\{p, q, r\} \in S_{m}$, and

$$
\begin{aligned}
b_{7, j}^{k, k, k} & =\frac{6\left(-j^{4}+3 k^{4}\right) \mu \sin ^{3}(k \pi / 2) \sin (j \pi / 2)}{\lambda \pi^{4}\left(j^{8}-10 j^{4} k^{4}+9 k^{8}\right)}, \\
b_{7, j}^{k, k, l} & =6 \mu \sin ^{2}(k \pi / 2) \sin (l \pi / 2) \sin (j \pi / 2) \\
& \times\left(j^{8}-2 j^{4} k^{4}-2 j^{4} l^{4}-6 k^{4} l^{4}+l^{8}+8 k^{8}\right) /\left[\left(\lambda \pi^{4}\left(l^{4}-j^{4}\right)\right.\right. \\
& \left.\times\left(-8 k^{4} l^{4}-8 j^{4} k^{4}+16 k^{8}+j^{8}-2 j^{4} l^{4}+l^{8}\right)\right], \\
b_{7, j}^{k, l, k} & =\frac{12 \mu \sin ^{2}(k \pi / 2) \sin (l \pi / 2) \sin (j \pi / 2)}{\lambda \pi^{4}\left(-8 k^{4} l^{4}-8 j^{4} k^{4}+16 k^{8}+j^{8}-2 j^{4} l^{4}+l^{8}\right)}, \\
b_{9, j}^{k, k, k} & =a_{8, j}^{k, k, k}=\frac{12 \mu \sin ^{3}(k \pi / 2) \sin (j \pi / 2)}{\lambda^{2} \pi^{8}\left(j^{8}-10 j^{4} k^{4}+9 k^{8}\right)}, \\
b_{9, j}^{k, k, l} & =\frac{\mu \sin ^{2}(k \pi / 2) \sin (l \pi / 2) \sin (j \pi / 2)\left(-l^{4}-3 j^{4}+4 k^{4}\right)}{\lambda^{2} \pi^{8}\left(l^{4}-j^{4}\right)\left(-8 k^{4} l^{4}-8 j^{4} k^{4}+16 k^{8}+j^{8}-2 j^{4} l^{4}+l^{8}\right)},
\end{aligned}
$$

with, again, similar relations when $k$ and $l$ are switched.

It can be noted by inspection of Equations (31), (33) and (18) that

$$
a_{6, j}^{k, k, k}=a_{6, j}^{k} \text { as obtained for the } k \text { th nonlinear normal mode, }
$$

and

$$
a_{8, j}^{k, k, k}=a_{8, j}^{k} \text { as obtained for the } k \text { th nonlinear normal mode. }
$$

Although it was noted earlier that the multi-mode manifold formulation should change these coefficients, these changes are due to the presence of quadratic nonlinearities which are not included in this example. Hence, for this system, the one-mode coefficients are the same for both the single- and multi-mode manifolds. Also, as a two-mode manifold was sought, it was not necessary to solve the three-mode subproblem in order to obtain a general solution.

Note that for this example system, as was the case for single-mode manifolds, the dynamics are obtained at order $N^{\prime}+2$ when the order of approximation of the multi-mode manifold is $N^{\prime}$. In general the dynamics are precise through order $N^{\prime}+Q-1$ when the lowest nonlinearity is of order $Q$. In the present case, the dynamics are obtained at fifth order by the coupled equations

$$
\left\{\begin{aligned}
\dot{u}_{k}= & v_{k}, \\
\dot{v}_{k}= & -\lambda(k \pi)^{4} u_{k}-2 \mu \sin (k \pi / 2)\left[\sum_{l \in S_{M}} u_{l} \sin (l \pi / 2)\right]^{3} \\
& -6 \mu \sin (k \pi / 2)\left[\sum_{l \in S_{M}} u_{l} \sin (l \pi / 2)\right]^{2}\left[\sum_{j \notin S_{M}} X_{j}^{(3)} \sin (j \pi / 2)\right]+\cdots,
\end{aligned}\right.
$$




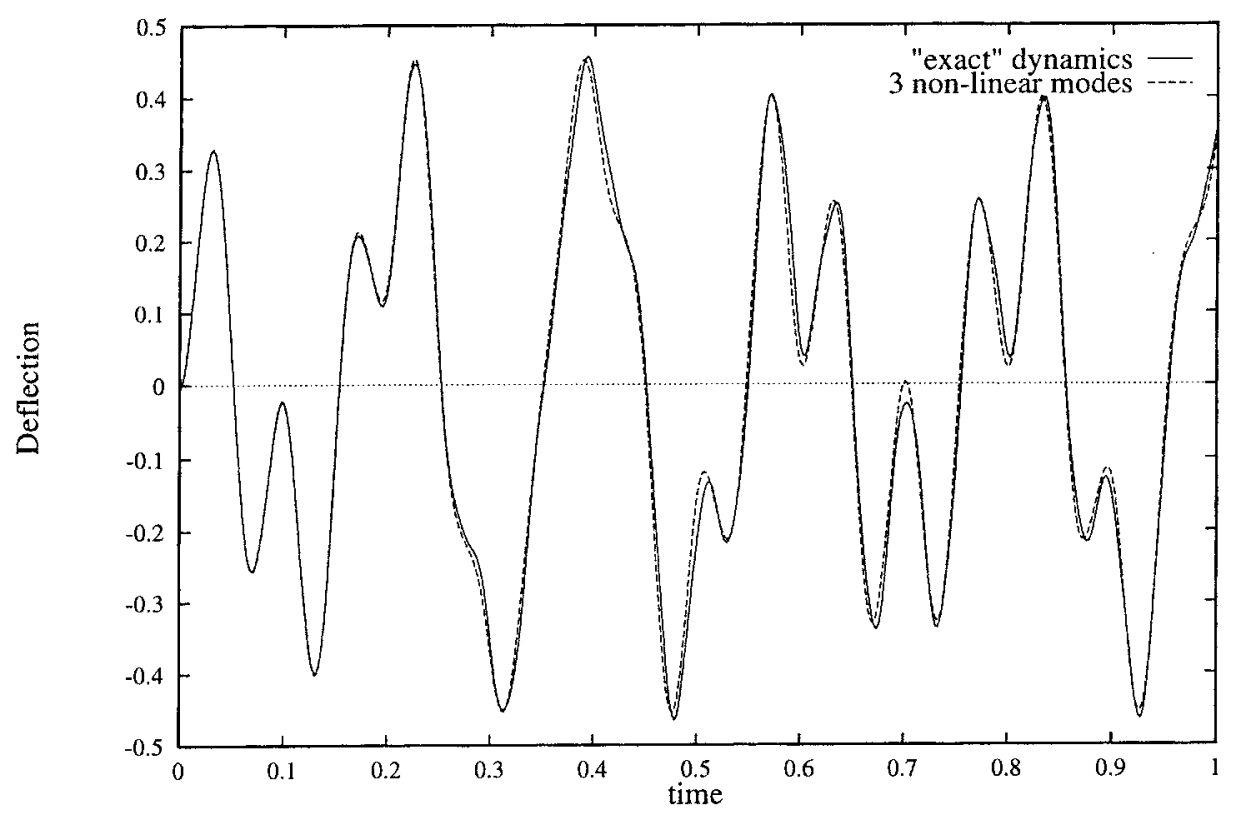

Figure 5. Deflection of the middle-point of the beam obtained by a third-order accurate three-mode invariant manifold. Initial conditions: $u_{1}(t=0)=u_{3}(t=0)=0.2, u_{2}(t=0)=0.1, v_{1}(t=0)=v_{2}(t=0)=v_{3}(t=0)=0$.

for $k \in S_{m}$, where $X_{j}^{(3)}$ represents the cubic part of $X_{j}$. Note that, in contrast with the single-mode manifold formulation, the dynamics of the various modeled nonlinear modes are coupled ( $u_{l}$ terms), so that essential interactions between them are allowed, while the coupling to the non-modeled modes has been removed, although their influence is included through the $X_{j}^{(3)}$ terms.

Results of simulations performed using either the above multi-mode manifold procedure or a linear modal analysis of the nonlinear system are shown in Figures 5 and 6 for a given set of initial conditions on a three-mode manifold, using the parameters $\lambda=1$, and $\mu=5000$. In these examples, the three-mode model is composed of the first three modes and can therefore be obtained directly from the two-mode model involving only the first and third modes, as all the coefficients corresponding to the added even mode vanish. Consistent with the theory, a given number of nonlinear modes embedded in the multi-mode manifold yields better results than the same number of linear modes used in a linear modal analysis procedure, all the more so as the influence of the nonlinearity increases.

\subsection{Finite-Element Based Example: A Pinned/Spring Euler-Bernoulli BEAM CONSTRAINED By A NONLINEAR TORSIONAL SPRING}

The computer program mentioned above was used for the analysis of the system shown in Figure 7. The finite element code PATRAN was used to create a beam model with 200 two-noded beam finite elements. The beam in Figure 7 is pinned at one end, with the other constrained with a linear spring, and a nonlinear torsional spring located at the pinned end. Once the linear modes of this model were known, the nonlinear torsional spring was added (analytically) to the pin location. The characteristics of the nonlinear spring and the linear eigenvectors were then used to construct the $[\alpha]$ and $[\beta]$ matrices. Using these, reduced equations for any chosen 


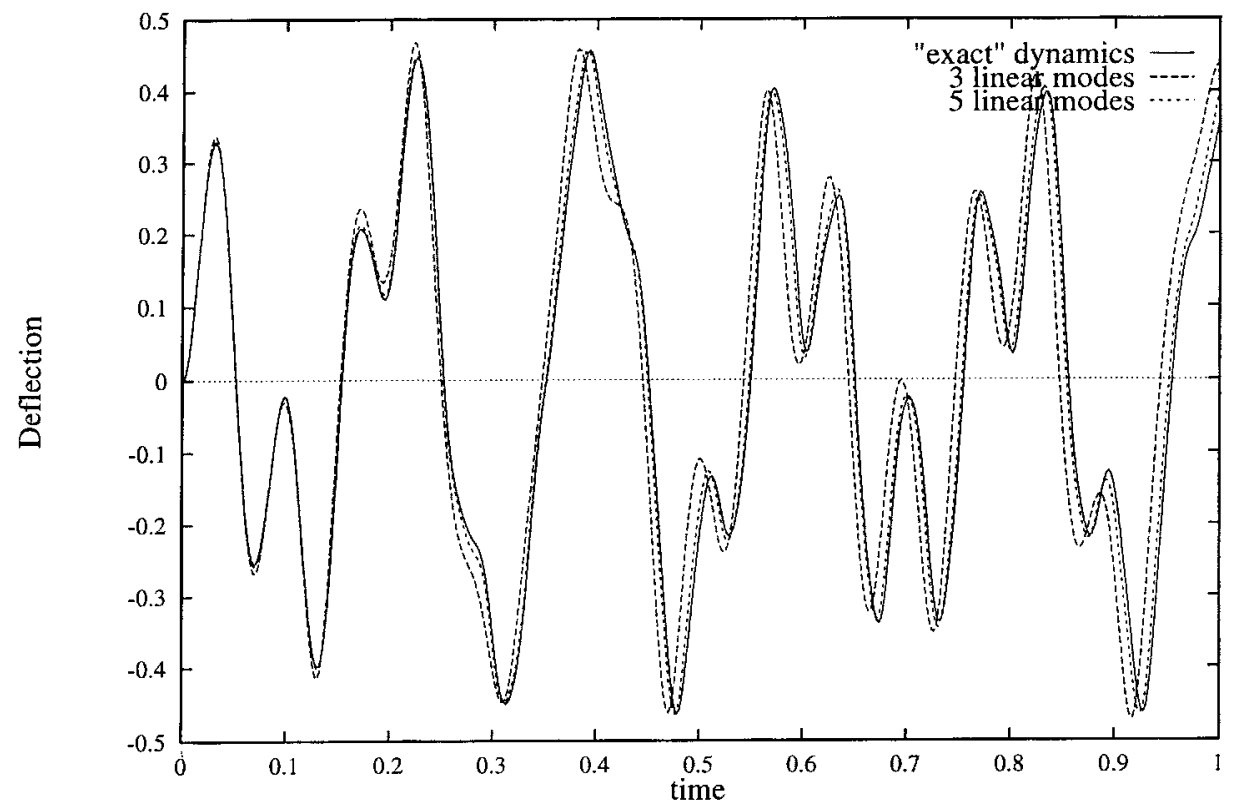

Figure 6. Deflection of the middle-point of the beam obtained by three-mode and five-mode linear modal analysis of the nonlinear system. Initial conditions: $u_{1}(t=0)=u_{3}(t=0)=0.2, u_{2}(t=0)=0.1, v_{1}(t=0)=v_{2}$ $(t=0)=v_{3}(t=0)=0$.

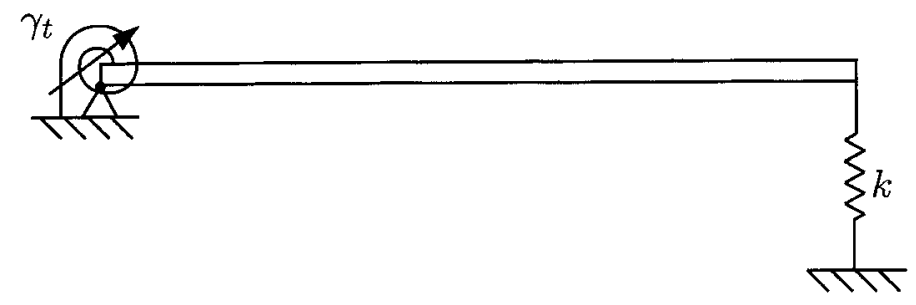

Figure 7. Schematic of finite element model: 200 beam elements were used to construct a beam with: $L=1 \mathrm{~m}$, $\rho=7860 \mathrm{~kg} / \mathrm{m}^{3}, E=2 \times 10^{11} \mathrm{~N} / \mathrm{m}^{2}, I=5 \times 10^{-8} \mathrm{~m}^{4}$. The parameters $k$ and $\gamma_{t}$ vary by case.

set of nonlinear normal modes may easily be generated. Once the reduced modal dynamics have been determined (here, through fourth-order Runge-Kutta numerical integration) the manifold coefficients may be used to reconstruct the non-modeled modal displacements, and the corresponding physical displacements. Two results are shown: in the first, the system is not internally resonant and the torsional spring contains both quadratic and cubic nonlinearities, and in the second, the linear spring is tuned such that a 2:1 internal resonance in generated between the second and third linear modes, and the torsional spring is purely quadratic.

The results for the first case, shown in Figure 8, illustrate the displacements at the beam's quarter span, predicted by an 'exact' solution ( 25 linear modes), a three-mode nonlinear manifold, and a three-linear-mode truncation. The first three modes were used in both three-mode models. For this case it can be seen that the linearly truncated model yields results which slowly diverge, while the other two match quite well. However, these small errors hardly seem to justify the effort necessary to generate the six-dimensional manifold, and the reduced equations it yields. One must keep in mind that the small errors shown here simply indicate 


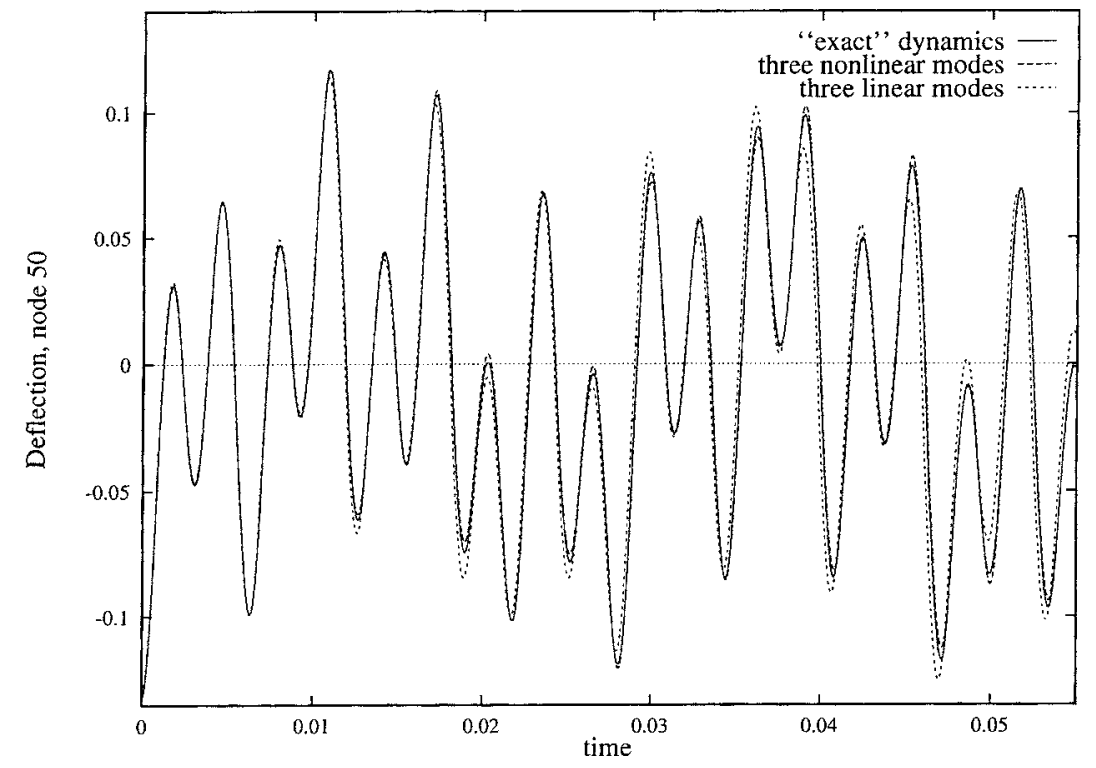

Figure 8. Deflection of node 50 (quarter span of beam) versus time using various methods. Here, $k=10^{8}$, and the nonlinear spring strength is $\gamma_{t}=5000 u^{\prime}(0)^{2}+20000 u^{\prime}(0)^{3}$, with initial conditions: $u_{1}(t=0)=-0.15$, $u_{2}(t=0)=0.12, u_{3}(t=0)=0.25, v_{1}(t=0)=v_{2}(t=0)=v_{3}(t=0)=0$.

that for this system, at this amplitude, with these initial conditions, the contributions of the non-modeled modes are not very significant. Changes in any of these parameters may affect the results in unexpected ways.

In the second case, Figure 9, the displacements at half span predicted by the 'exact' solution, the two-mode manifold, and a two-linear-mode truncation are shown. Both two-mode solutions use the second and third (internally resonant) linear modes. Here, a significant error, in the form of a nearly constant offset, is present from the outset. Analysis shows that this offset is due to contributions from the first mode, a phenomenon which deserves some exploration. The two-mode manifold solution explicitly simulates the second and third modes, and then assembles the contributions of the remaining modes, including the first, to obtain the dynamics of the entire system. Hence, the obtained offset is a polynomial function of $u_{2}, u_{3}, v_{2}$, and $v_{3}$. The 'exact' solution is initiated on the manifold, so the initial conditions in the second and third modes, together with the manifold coefficients, specify the initial conditions of all the modes, which are then simulated. For this solution, all 25 modes interact dynamically to maintain the offset. The two-mode truncation simply assumes that all other modes remain quiescent and do not contribute, an approach with no hope of yielding accurate results in this situation. For this case it is apparent that modes two and three are not easily separable from the system. That is, that mode one (at least!) must be accounted for or contamination is ensured. Also, the manifold solution reproduces the 'exact' one exceptionally well, indicating considerable manifold accuracy.

The two examples above illustrate that, while exceptional results are not guaranteed, important (and unexpected) effects, when present, may be captured. Indeed, it is these unpredictable modal interactions which prove the utility of this approach for large scale systems. Due to its finite element origin, and the systematic reduction procedure, the above example could just as 


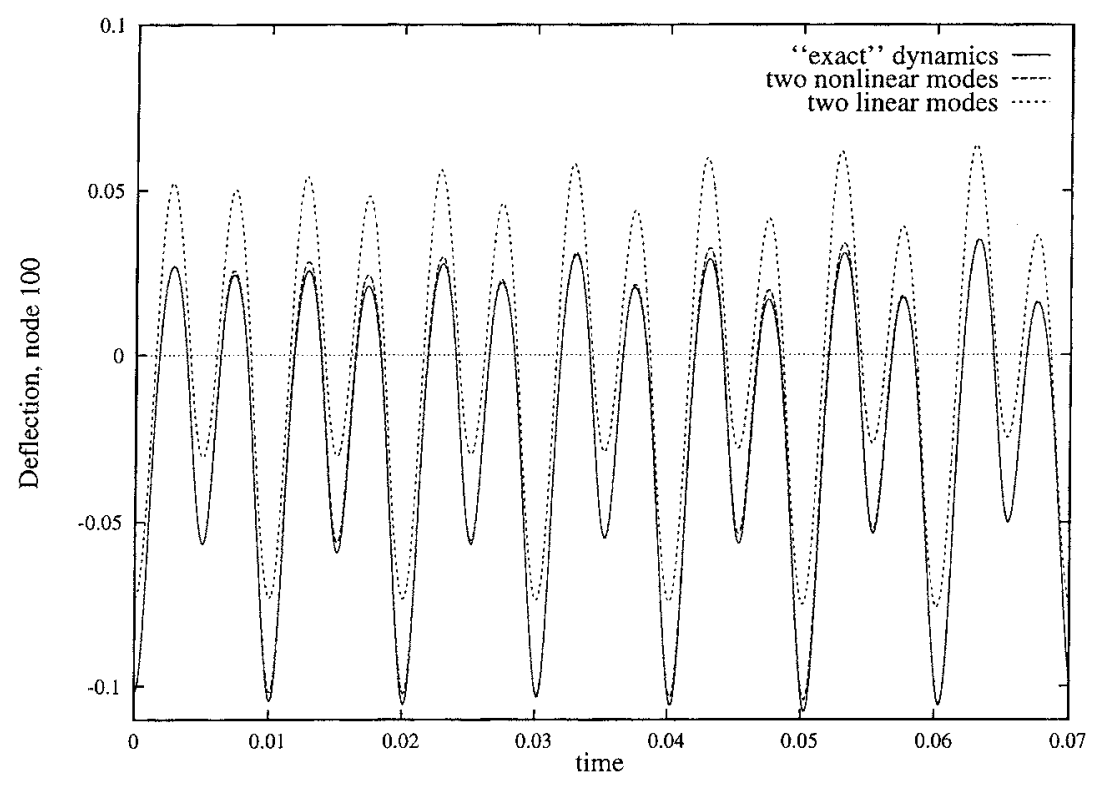

Figure 9. Deflection of node 100 (half span of beam) versus time using various methods. Here, $k=1.185 \times 10^{6}$, and the nonlinear spring strength is $\gamma_{t}=10000 u^{\prime}(0)^{2}$, with initial conditions: $u_{2}(t=0)=0.2, u_{3}(t=0)$ $=-0.35, v_{2}(t=0)=v_{3}(t=0)=0$.

easily have been a plate, shell, or general structure, and the reduction procedure would remain unchanged. As such, though the reduction process is analytically complex, its automation enables the generation of rigorous, uncontaminated, reduced-order models of a large variety of nonlinear structural systems.

\section{Conclusions}

The developments in Sections 2 and 3 suggest that the concept of invariant manifolds has potentially important implications for nonlinear structural dynamics problems. This is a powerful way of defining a nonlinear modal analysis for nonlinear systems, in the sense that proper interaction between the various modeled modes is allowed and accounted for, while contamination with the non-modeled modes is eliminated. This property, which is essential for proper simulation of the dynamics of a system once its most important modes have been selected, is an extension to nonlinear systems from the modal analysis that exists for linear systems.

In addition, when the original equations of motion are given in terms of the linear modal coordinates, each nonlinear mode or multi-mode manifold contains, as its kernel, the corresponding system in which non-modeled modes are entirely ignored. Hence, in regions where the manifold description is accurate, results are guaranteed to be more precise than those produced through simple truncation, as the reduction procedure embeds the influence of additional, non-modeled, modes.

The utility of this approach is enhanced by the fact that the coefficients are generated through linear subproblems, and furthermore by the generalizations which enable additional reduction and analytical solutions. The advent of powerful symbolic manipulation packages has enabled the generation of these solutions for some restricted classes of systems (at least). 
Though the solutions outlined herein do not account for all possible nonlinear terms, a considerable subset of structural systems are included. Thus, the generation of rigorous, reducedorder models for a wide variety of nonlinear systems is reduced to a subroutine.

Illustrative results are provided for both an analytically manageable system, and a more complex, finite-element based system. The analytical results depict typical expressions for the manifold expansion coefficients and the corresponding reduced equations of motion, while the finite-element based system emphasizes that practical application is not limited to highly idealized, analytically tractable systems. Numerical integration results are provided for both systems as an indication of the accuracy and utility of the approach.

The methods proposed herein to generate reduced-order models have potentially important implications for many areas, including structural dynamics and control, where accurate loworder models are of interest.

\section{Appendix A: Additional Formulas}

The constants from the two and three mode problems are as follows:

$$
\begin{aligned}
C_{1, i}^{3, k, l}= & 2 \sum_{q \in S_{M}} \alpha_{k, k}^{q} a_{5, i}^{q, l}, \\
C_{2, i}^{3, k, l}= & 2 \sum_{q \in S_{M}} \alpha_{k, l}^{q} a_{5, i}^{q, k}, \\
C_{7, i}^{3, k, l}= & \beta_{k, k, l}^{i}+\sum_{q \notin S_{M}}\left(2 \alpha_{k, q}^{i} a_{3, q}^{k, l}+\alpha_{l, q}^{i} a_{3, q}^{k, k}\right)-\sum_{q \in S_{M}}\left(\alpha_{k, k}^{q} b_{4, i}^{l, q}+\alpha_{k, l}^{q} b_{4, i}^{k, q}\right), \\
C_{5, i}^{3, k, l}= & 2 \sum_{q \notin S_{M}} \alpha_{k, q}^{i} a_{5, q}^{k, l}, \\
C_{6, i}^{3, k, l}= & \sum_{q \notin S_{M}} \alpha_{l, q}^{i} a_{5, q}^{k, k}, \\
C_{8, i}^{3, k, l, m}= & 2 \sum_{q \in S_{M}} \alpha_{k, l}^{q} a_{5, i}^{q, m}, \\
C_{9, i}^{3, k, l, m}= & \sum_{q \notin S_{M}}\left(\alpha_{k, q}^{i} a_{3, q}^{l, m}+\alpha_{m, q}^{i} a_{3, q}^{k, l}+\alpha_{l, q}^{i} a_{3, q}^{m, k}\right) \\
& +\frac{1}{2} \sum_{q \in S_{M}}\left(\alpha_{m, k}^{q} b_{4, i}^{l, q}+\alpha_{l, k}^{q} b_{4, i}^{m, q}+\alpha_{m, l}^{q} b_{4, i}^{k, q}\right)+\frac{1}{2} \beta_{m, l, k}^{i}, \\
C_{10, i}^{3, k, l, m}= & 2 \sum_{q \notin S_{M}} \alpha_{k, q}^{i} a_{5, q}^{l, m} .
\end{aligned}
$$

\section{Acknowledgment}

This work has been supported by a grant from the Army Research Office, under Program Manager Dr. Gary L. Anderson. 


\section{References}

1. Meirovitch, L., Analytical Methods in Vibrations, MacMillan, New York, 1967.

2. Nayfeh, A. H., Chin, C., and Nayfeh, S. A., 'On nonlinear normal modes of systems with internal resonance', ASME, Journal of Vibration and Acoustics 118, 1996, 340-345.

3. Rosenberg, R. M., 'On nonlinear vibrations of systems with many degrees of freedom', Advances in Applied Mechanics 9, 1966, 155-242.

4. Aubrecht, J. and Vakakis, A. F., 'Localized and non-localized nonlinear normal modes in a multi-span beam with geometric nonlinearities', ASME, Journal of Applied Mechanics 118, 1996, 533-542.

5. Chakraborty, G., Mallik, A. K., and Hatwal, H., 'Normal modes and near-resonance response of beams with non-linear effects', Journal of Sound and Vibration 210(1), 1998, 19-36.

6. Shaw, S. W. and Pierre, C., 'Normal modes for non-linear vibratory systems', Journal of Sound and Vibration 164(1), 1993, 85-124.

7. Shaw, S. W. and Pierre, C., 'Normal modes of vibration for non-linear continuous systems', Journal of Sound and Vibration 169(3), 1994, 319-347.

8. Shaw, S. W., 'An invariant approach to nonlinear normal modes of vibration', Journal of Nonlinear Science 4, 1994, 419-448.

9. Carr, J., Applications of Center Manifold Theory, Springer-Verlag, Berlin, 1981.

10. Boivin, N., Pierre, C., and Shaw, S. W., 'Non-linear normal modes, invariance, and modal dynamics approximations of non-linear systems', Nonlinear Dynamics 8, 1995, 315-346.

11. King, M. E. and Vakakis, A. F., 'An energy based formulation for computing nonlinear normal modes in undamped continuous systems', ASME, Journal of Vibration and Acoustics 116, 1994, 332-340.

12. Chen, S.-L. and Shaw, S. W., 'Normal modes for piecewise linear vibratory systems', Nonlinear Dynamics 10, 1996, 135-164.

13. Slater, J. C., 'A numerical method for determining nonlinear normal modes', Nonlinear Dynamics 10, 1996, 19-30.

14. Burton, T. B. and Hamdan, M. N., 'On the calculation of non-linear normal modes in continuous systems', Journal of Sound and Vibration 197(1), 1996, 117-130.

15. Slater, J. C. and Inman, D. J., 'On the effect of weak non-linearities on linear controllability and observability norms, an invariant manifold approach', Journal of Sound and Vibration 199(3), 1997, 417-429.

16. Qasi, M. I., 'Nonlinear normal modes of a lumped parameter system', Journal of Sound and Vibration 205(2), 1997, 205-211.

17. King, M. E. and Vakakis, A. F., 'An energy based approach to computing resonant nonlinear normal modes', ASME, Journal of Applied Mechanics 63, 1996, 810-819.

18. Leung, A. Y. T. and Ge, T., 'Normal multi-modes of non-linear Euler beams', Journal of Sound and Vibration 202(2), 1997, 145-160.

19. Pellicano, F. and Mastroddi, F., 'Applicability conditions of a non-linear superposition technique', Journal of Sound and Vibration 200(1), 1997, 3-14.

20. Boivin, N., Pierre, C., and Shaw, S. W., 'Non-linear modal analysis of structural systems featuring internal resonances', Journal of Sound and Vibration 182(2), 1995, 336-341.

21. Boivin, N., Pierre, C., and Shaw, S. W., 'Non-linear modal analysis of structural systems using multi-mode invariant manifolds', in Proceedings of the AIAA Dynamics Specialists Conference, Hilton Head, SC, 1994, Paper No. 94-1672.

22. Boivin, N., 'Non-linear modal analysis of structural systems using invariant manifolds', Ph.D. Thesis, The University of Michigan, Ann Arbor, MI, 1995. 